\title{
Intradural Extramedullary Epithelioid Hemangioendothelioma of the Thoracic Spinal Cord: A Case Report
}

\author{
Munenari IKEZAWA, ${ }^{1,2}$ Masashi FuJIMOTO, ${ }^{2}$ Hirofumi NiSHIKAWA, ${ }^{1}$ \\ Satoru TANIOKA, ${ }^{3}$ Yusuke KURODA, ${ }^{2}$ Yume SuZUKI, ${ }^{2}$ \\ Masaki MIZUNO, ${ }^{1,4}$ and Hidenori SUZUKI ${ }^{2}$
}

\author{
${ }^{1}$ Department of Neurosurgery, Suzuka Kaisei Hospital, Suzuka, Mie, Japan \\ ${ }^{2}$ Department of Neurosurgery, Mie University Graduate School of Medicine, Tsu, Mie, Japan \\ ${ }^{3}$ Department of Neurosurgery, Mie Chuo Medical Center, Tsu, Mie, Japan \\ ${ }^{4}$ Department of Minimum-Invasive Neurospinal Surgery, Mie University Graduate School of \\ Medicine, Tsu, Mie, Japan
}

\begin{abstract}
Epithelioid hemangioendothelioma (EHE) is a rare vascular tumor, and develops infrequently in the central nervous system. To our knowledge, this is the first case of EHE of the spinal cord. An 85-year-old man presented with about 6-month progressive myelopathy. Magnetic resonance imaging (MRI) demonstrated an oval-shaped intradural extramedullary mass at T10 level with extensive intramedullary edema. A reddish tumor was removed via a total laminectomy of T9-T10. Histologically, the tumor cells with nuclear atypia and active mitoses were immunopositive for vascular tumor markers, and formed a lobular architecture associated with capillary-sized vessels lined with edematous endothelial cells. Although very rare, EHE should be considered in the differential diagnosis of a spinal intradural extramedullary tumor.
\end{abstract}

Keywords: epithelioid hemangioendothelioma, spinal cord, vascular tumor

\section{Introduction}

Epithelioid hemangioendothelioma (EHE) is a rare vascular tumor, accounting for less than $1 \%$ of all vascular tumors., ${ }^{1,2)}$ EHE is most often found in the lung, liver, and bones, and is rarely located in the skull base, dura, or brain parenchyma. ${ }^{1,2)}$ To date, no previous reports have described EHE of the spinal cord, although there was a report of EHE of the filum terminale. ${ }^{3)}$ The authors here report the first case of EHE of the thoracic spinal cord.

Received September 23, 2020; Accepted December 7, 2020

Copyright $@ 2021$ The Japan Neurosurgical Society This work is licensed under a Creative Commons AttributionNonCommercial-NoDerivatives International License.

\section{Case Report}

An 85-year-old man with a past history of cerebral infarction and the sequela, mild right hemiparesis, presented with progressive gait disturbance, hypesthesia in the lower limbs, and bladder bowel dysfunction for the last 6 months. On the neurological examination, his muscle power in the right and left lower limbs was $3 / 5$ and $1 / 5$ in manual muscle testing, respectively. Sensation to pinprick, temperature, and light touch was reduced at the level of T10 and below. Both lower limbs showed hyporeflexia possibly by aging. Magnetic resonance imaging (MRI) showed an intradural extramedullary mass lesion at T10 level, which was oval-shaped and well-defined; was isointense on T1-weighted images and hyperintense on T2-weighted images; and had an intense peripheral ring enhancement with homogenous central enhancement by a contrast 

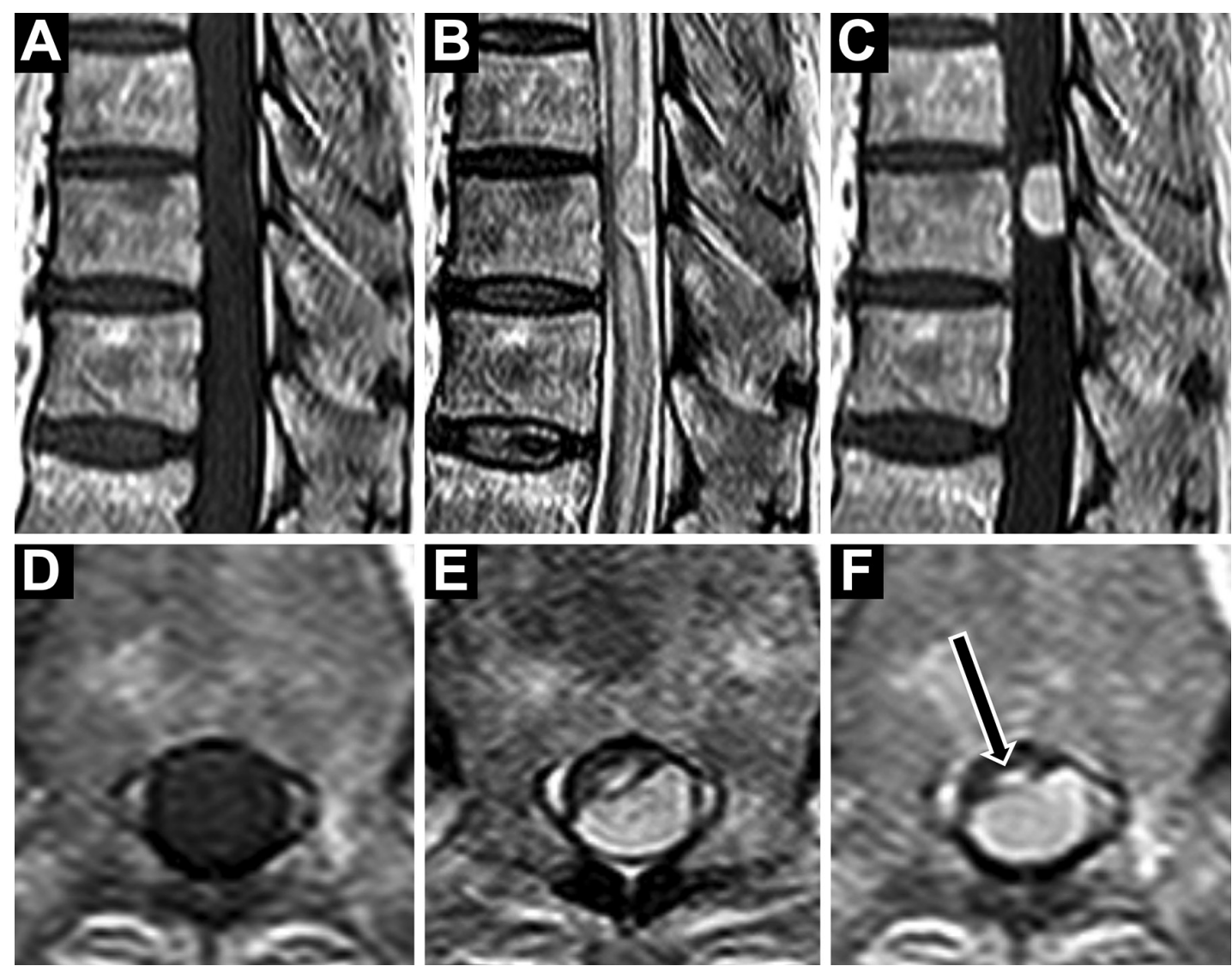

Fig. 1 Preoperative spinal MRI (A-C, sagittal images; D-F, axial images). T1-weighted images (A, D) show an intradural extramedullary oval-shaped isointense lesion at T10 level, which is hyperintense on T2-weighted images (B, E) and has an intense peripheral ring enhancement with homogenous central enhancement by gadolinium (C, F). An extensive intramedullary hyperintense area is also revealed on T2-weighted images (B, E), and the part of the area in the spinal cord is enhanced at T10 level (arrow; F). MRI: magnetic resonance imaging.

medium (Fig. 1). T2-weighted MRI also revealed an intramedullary hyperintense area extending from T4 level to conus medullaris, which was partially enhanced at T10 level. There were no findings suggesting metastatic lesions on whole-body computed tomography scans. The tumor was diagnosed as neurinoma or meningioma tentatively, and removed surgically as follows.

Under general anesthesia, the patient was set in a prone position, and a midline skin incision was made from the spinous process of T8 to that of T10. Under a microscopic magnification, when total laminectomy was performed at T9-10 and the dura mater was opened, a reddish mass and dilated veins were revealed on the thoracic spinal cord and under the arachnoid membrane (Fig. 2). The arachnoid membrane was separated easily from the tumor. The tumor was partially adhered to the spinal cord, but existed only extra-pially. The tumor was totally removed en bloc. No feeding arteries were found while removing the tumor.
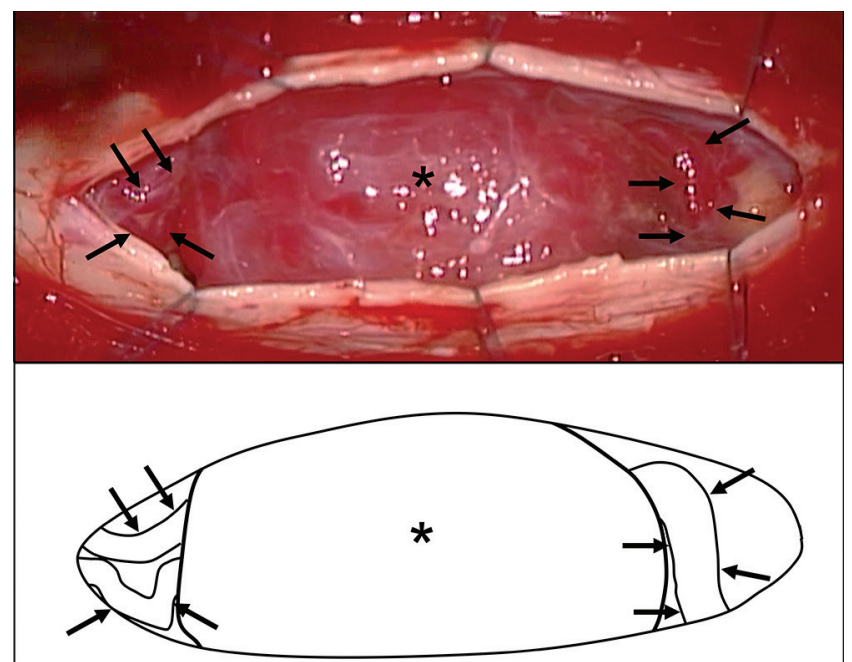

Fig. 2 An intraoperative photograph through the surgical microscope (upper) and the illustration (lower). After dural opening, a reddish mass (asterisk) and some dilated veins (arrows) are found on the thoracic spinal cord. 


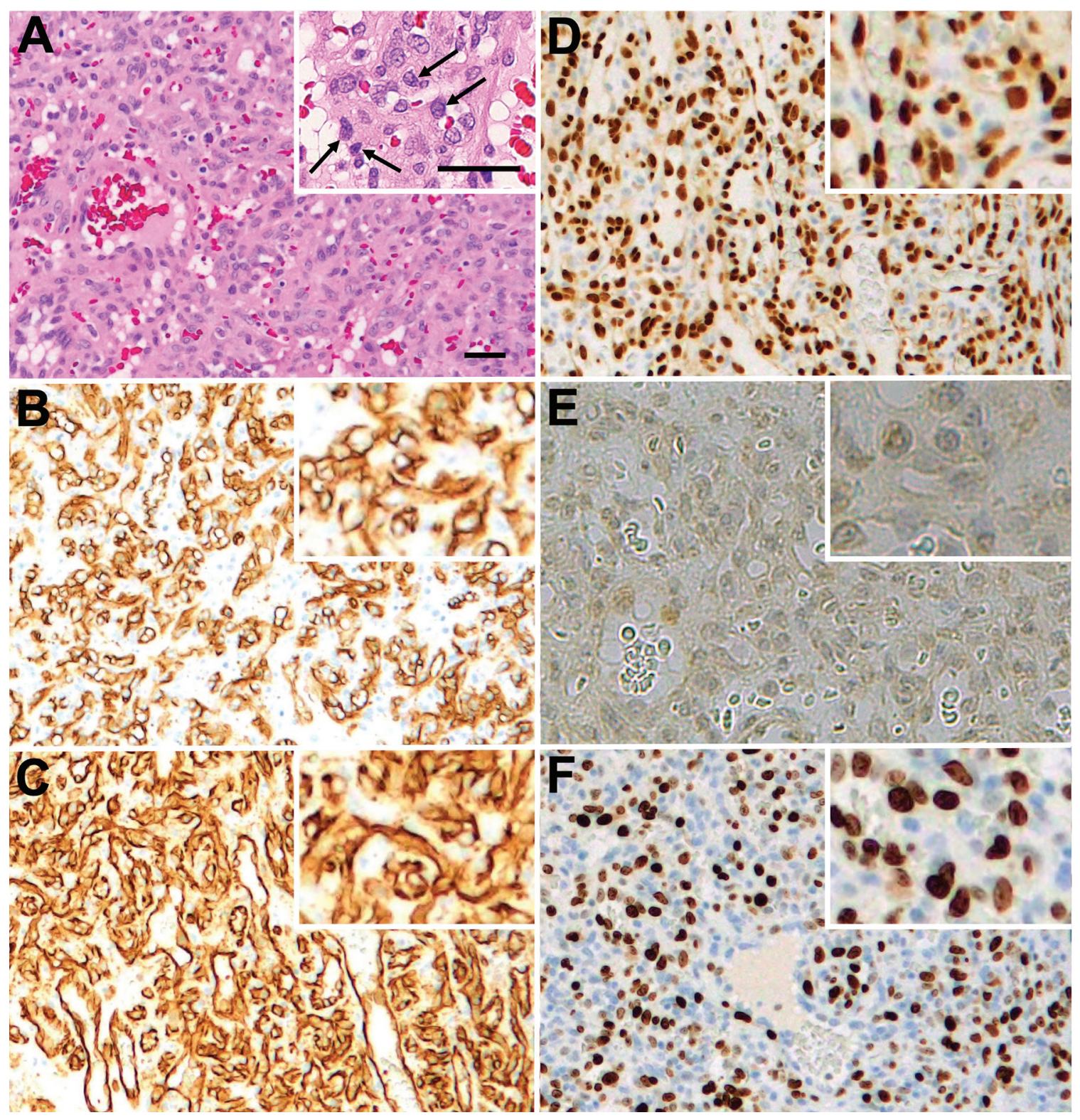

Fig. 3 Histopathological findings. Hematoxylin and eosin staining (A) shows many capillary-sized vessels lined by endothelial cells and tumor cells with mitoses (arrows). Immunostaining shows that endothelial cells and tumor cells are positive for CD31 (B) and CD34 (C), and that nuclei of tumor cells are positive for ERG (D) and negative for STAT6 (E). MIB-1 index is approximately $32.6 \%$ (F). Scale bar $=25 \mu \mathrm{m}$. ERG: erythroblast-transformationspecific-related gene.

On histopathological examinations, tumor cells had oval nuclei with frequent atypia as well as mitoses and pale eosinophilic cytoplasm, forming lobular architectures associated with capillary-sized vessels (Fig. 3A). Endothelial cells lining vessels were edematous. Immunostaining showed that endothelial cells and tumor cells were positive for factors CD31 (Fig. 3B) and CD34 (Fig. 3C), and that nuclei of cells were positive for erythroblast-transformation-specific-related gene (ERG) (Fig. 3D). However, tumor cells were negative for signal transducer and activator of transcription 6 (STAT6) (Fig. 3E). MIB-1 index was approximately $32.6 \%$ (Fig. 3F).

The postoperative course was uneventful. His motor disturbance gradually improved, although sensory disturbances in the lower limbs persisted. MRI obtained 2 weeks post-surgery showed no residual tumor and the reduction of an hyperintense area on T2-wegihted images to between T6 and T10 levels. The patient and his family refused any adjuvant therapy including chemotherapy and 
radiotherapy because of his old age. The patient was transferred to a rehabilitation center on the 21st postoperative day, but thereafter his neurological status was not improved. He underwent neither adjuvant therapy nor neuroimaging, and died of aspiration pneumonia 19 months after surgery.

\section{Discussion}

Hemangioendothelioma is a vascular tumor composed of endothelial-derived tumor cells, and has been used to designate a heterogeneous group of vascular lesions ranging from benign conventional hemangioma to angiosarcoma. ${ }^{1)} \mathrm{EHE}$ is one of the subtypes of hemangioendotheliomas. EHE develops from vascular endothelial or pre-endothelial cells, and is the second malignant vascular tumor following angiosarcoma according to the International Society for the Study of Vascular Anomalies classification for vascular anomalies, ${ }^{4)}$ accounting for less than $1 \%$ of all vascular tumors. ${ }^{1)}$ EHE may develop at any age, but is the most popular in middle-aged people. ${ }^{5)}$ EHE can arise in diverse organs and tissues including soft tissues, lungs, liver, bones, breasts, lymph nodes, and many other sites, while it has been rarely reported in the skull base, dura, or brain parenchyma. ${ }^{1,6-8)}$ To the best of our knowledge, there has been no report of the primary EHE of the spinal cord, although there is only one case report of filum terminale EHE. ${ }^{3)}$ The EHE of the filum terminale was isointense on both $\mathrm{T} 1$ and $\mathrm{T} 2$ sequences of MRI, and was enhanced intensely by a contrast medium. ${ }^{3)}$ The present case was the first EHE case of the spinal cord, which was an extra-pial tumor with isointensity on T1-weighted images, hyperintensity on T2-weighted images, and central contrast enhancement with more intense enhancement peripherally. Our case was also associated with an extensive intramedullary edema, which was partially enhanced possibly by blood-brain barrier disruption.

The differential diagnosis of intradural extramedullary EHE includes neurinoma, meningioma, and other vascular tumors. Preoperative diagnosis of EHE is challenging because of its rarity and no characteristic findings on neuroimages. The most common intradural extramedullary tumors are neurinomas and meningiomas, both of which are usually hypo- to iso-intense on T1-weighted images, iso- to hyper-intense on T2-weighted images, and are enhanced well. ${ }^{9,10}$ If neurinomas or meningiomas have cystic changes, necrosis, or calcification on neuroimages, they can be differentiated from vascular tumors such as hemangioma and EHE; otherwise, it is almost impossible to do that. The differentiation among vascular tumors including extramedullary hemangioma and EHE may be also impossible on neuroimages. ${ }^{3,11)}$

Histologic and cytologic features together with immunohistochemistry analyses allow us to differentiate EHE from hemangioma and angiosarcoma. ${ }^{1)}$ To diagnose epithelioid hemangioma, areas of conventional epithelioid hemangioma morphology should be sought: conventional epithelioid hemangioma is comprised of vessels lined by plump endothelial cells that project into vascular lumina, causing the so-called tombstoning. ${ }^{12)}$ In contrast, EHE is incapable of forming true vascular channels, although EHE shows endothelial differentiation and consists of endothelial cells arranged in nests and cords, associated with spindle-shaped tumor cells. ${ }^{13,14)}$ Murali et al. ${ }^{14)}$ reported the cytologic characteristics of EHE, which consists of epithelioid cells with moderate to large amounts of cytoplasm as well as nuclear pleomorphism associated with nuclear grooves. ${ }^{14)}$ As to immunohistochemistry, CD34 is poorly specific for vascular tumors and positive in more than $90 \%$ of vascular tumors and a variety of mesenchymal tumors, whereas CD31 and ERG are more specific vascular tumor markers than CD34. ${ }^{15,16)}$ As ERG is a nuclear stain, it complements the cytoplasmic and membranous staining of CD31. ${ }^{16)}$ EHE is positive for both CD31 and ERG, confirming the tumor's endothelial nature. ${ }^{13)}$ STAT6 is a highly sensitive marker of solitary fibrous tumors and hemangiopericytomas. ${ }^{17)}$ In this case, the tumor was positive for CD31, CD34, and ERG, but negative for STAT6, indicating a benign or malignant vascular tumor, ${ }^{16)}$ while denying solitary fibrous tumor and hemangiopericytoma. Angiosarcoma was excluded because of no well-formed vascular channels in this case, although very high MIB-1 index indicated that the tumor cells were mitotically active like angiosarcoma. ${ }^{18)}$ Hemangioma was also excluded based on very high MIB-1 index and no areas of the so-called tombstoning. The tumor in this case might arise from blood vessels on the pial surface of the spinal cord because the tumor existed extra-pially and was easily separated from the arachnoid membrane.

Due to its rarity, treatment options and the prognosis of EHE are controversial. EHE has no standard treatments, and has been treated by surgery only, or chemotherapy and/or radiation therapy following surgery. ${ }^{1)}$ For localized lesions, surgical resection would be better. ${ }^{1,19)}$ There is no consensus about the efficacy of radiation therapy. ${ }^{1)}$ In contrast to hemangioma, however, EHE has metastatic potential. ${ }^{1)}$ Case reports and case series have reported the efficacy of cytotoxic chemotherapy, immune therapy, and targeted therapies for metastatic diseases of EHE, ${ }^{19)}$ although no data of large randomized controlled trials are 
available as to the optimal treatment strategy. Reported chemotherapy regimens include (1) bevacizumab monotherapy; carboplatin, pemetrexed, and bevacizumab; carboplatin and etoposide; and adriamycin, dacarbazine, and ifosfamide for cytotoxic chemotherapy; (2) interferon alpha; and lenalidomide for immune therapy; (3) apatinib; sorafenib; pazopanib; and sirolimus for targeted therapies; and (4) combined chemotherapy such as apatinib combined with doxorubicin and cyclophosphamide. ${ }^{19,20)}$ In this case, surgical resection of intradural extramedullary EHE was effective for improving the patient's neurological findings, but a long-term outcome was poor without adjuvant therapies.

Although EHE is very rare, especially in the spinal cord, we should consider it in the differential diagnosis of a well-demarcated and well-enhanced mass lesion in the intradural extramedullary space of the spine.

\section{Conflicts of Interest Disclosure}

There are no conflicts of interest and nothing to disclose.

\section{References}

1) Sardaro A, Bardoscia L, Petruzzelli MF, Portaluri M: Epithelioid hemangioendothelioma: an overview and update on a rare vascular tumor. Oncol Rev 8: 259,2014

2) Omerhodžić I, Bilalović N, Rovčanin B, et al.: Primary epithelioid hemangioendothelioma in the cerebellum: case report with reference to drastic change in the who classification. Acta Clin Croat 57: 570-576, 2018

3) Marucci G, Barbanera A, Tosi AL, et al.: Epithelioid hemangioendothelioma of the spinal cord: description of a case with cytogenetic analysis. Int J Surg Pathol 14: 340-343, 2006

4) Dasgupta R, Fishman SJ: ISSVA classification. Semin Pediatr Surg 23: 158-161, 2014

5) Schattenberg T, Kam R, Klopp M, et al.: Pulmonary epithelioid hemangioendothelioma: report of three cases. Surg Today 38: 844-849, 2008

6) Chow LT, Chow WH, Fong DT: Epithelioid hemangioendothelioma of the brain. Am J Surg Pathol 16: 619-625, 1992

7) Nora FE, Scheithauer BW: Primary epithelioid hemangioendothelioma of the brain. Am J Surg Pathol 20: 707-714, 1996
8) Taratuto AL, Zurbriggen G, Sevlever G, Saccoliti M: Epithelioid hemangioendothelioma of the central nervous system. Immunohistochemical and ultrastructural observations of a pediatric case. Pediatr Neurosci 14: 11-14, 1988

9) Choi BY, Chang KH, Choe G, et al.: Spinal intradural extramedullary capillary hemangioma: MR imaging findings. AJNR Am J Neuroradiol 22: 799-802, 2001

10) Chung SK, Nam TK, Park SW, Hwang SN: Capillary hemangioma of the thoracic spinal cord. $J$ Korean Neurosurg Soc 48: 272-275, 2010

11) Kaneko Y, Yamabe K, Abe M: Rapid regrowth of a capillary hemangioma of the thoracic spinal cord. Neurol Med Chir 52: 665-669, 2012

12) Papke DJ, Hornick JL: What is new in endothelial neoplasia? Virchows Arch 476: 17-28, 2020

13) Hornick JL: Limited biopsies of soft tissue tumors: the contemporary role of immunohistochemistry and molecular diagnostics. Mod Pathol 32: 27-37, 2019

14) Murali R, Zarka MA, Ocal IT, Tazelaar HD: Cytologic features of epithelioid hemangioendothelioma. Am J Clin Pathol 136: 739-746, 2011

15) Gill R, O’Donnell RJ, Horvai A: Utility of immunohistochemistry for endothelial markers in distinguishing epithelioid hemangioendothelioma from carcinoma metastatic to bone. Arch Pathol Lab Med 133: 967-972, 2009

16) Sullivan HC, Edgar MA, Cohen C, Kovach CK, HooKim K, Reid MD: The utility of ERG, CD31 and CD34 in the cytological diagnosis of angiosarcoma: an analysis of 25 cases. J Clin Pathol 68: 44-50, 2015

17) Han $Y$, Zhang $Q$, Yu X, et al.: Immunohistochemical detection of STAT6, CD34, CD99 and BCL-2 for diagnosing solitary fibrous tumors/hemangiopericytomas. Int J Clin Exp Pathol 8: 13166-13175, 2015

18) Lin MS, Ngo T, Schwartz MR, Mehta RR, Ayala AG, Ro JY: Anastomosing hemangioma of the breast: an unusual case at an unusual site. J Breast Cancer 23: e15, 2020

19) Rosenberg A, Agulnik M: Epithelioid hemangioendothelioma: update on diagnosis and treatment. Curr Treat Options Oncol 19: 19, 2018

20) Zhang XQ, Chen H, Song S, Qin Y, Cai LM, Zhang F: Effective combined therapy for pulmonary epithelioid hemangioendothelioma: a case report. World $J$ Clin Cases 8: 2009-2015, 2020

Corresponding author: Munenari Ikezawa, MD Department of Neurosurgery, Suzuka Kaisei Hospital, 112-1 Kokufucho, Suzuka, Mie 513-8505, Japan. e-mail: munenari629@gmail.com 